\title{
APPROXIMATING ZARISKI DECOMPOSITION OF BIG LINE BUNDLES
}

\author{
By TAKAO FUJiTa
}

In this note we prove the following

THEOREM. Let $L$ be a line bundle on a variety $V$ with $n=\operatorname{dim} V$. Suppose that $h^{0}(V, t L) \geqq d t^{n} / n !+\boldsymbol{\phi}(t)$ for infinitely many positive integers $t$, where $\phi(t)$ is a function with $\lim _{t \rightarrow \infty} \phi(t) / t^{n}=0$ and $d$ is a positive real number. Then, for any $\boldsymbol{\varepsilon}>0$, there is a birational morphism $\pi: M \rightarrow V$ together with an effective $\boldsymbol{Q}$-divisor $E$ on $M$ such that $H=\pi^{*} L-E$ is a semiample $\boldsymbol{Q}$-bundle with $H^{n}>d-\varepsilon$.

If $H^{0}\left(t \pi^{*} L\right)=H^{0}(t H)$ for every $t>0$ such that $t E$ is a $Z$-divisor, then $\pi^{*} L=$ $E+H$ is a Zariski decomposition of $L$. In such a case we have $h^{0}(V, t L)=$ $d t^{n} / n !+\left(\right.$ lower order terms) for $d=H^{n}$. However, unfortunately, such a decomposition does not always exist. Our result can be viewed as an approximation of Zariski decomposition.

Combined with other techniques, our theorem can be used to derive various inequalities concerning intersection numbers of line bundles. In many cases it is almost as powerful as the theory of singular Hermitian metrics (cf. [D1], [D2]) or the theory of analytic Zariski decomposition (cf. [T]). Details of such applications will be discussed elsewhere.

For the proof of the theorem, we use the following

LEMma 1. Let $L$ be a line bundle on $V$. Suppose that $H=L-E+G$ is nef and big $\boldsymbol{Q}$-bundle for some effective $\boldsymbol{Q}$-divisor $E, G$ such that $H^{n-1} E=0$. Then any component of $E$ is a fixed component of $|L|$.

Proof. The following argument is just a variant of $[\mathrm{F} ;(2.5)]$. Replacing $V$ by some birational model and pulling thing back if necessary, we can assume that $|L|=F+M$ with Bs $M=\emptyset$, where $F$ is the fixed part of $|L|$. It suffices to derive a contradiction assuming that $G+F-E$ is not effective as a $\boldsymbol{Q}$-divisor.

Take an effective $\boldsymbol{Q}$-divisor $D$ such that $H-\varepsilon D$ is ample for any small $\varepsilon>0$. Take small enough $\varepsilon$ such that $\varepsilon D+G+F-E$ is not effective. Since $L-F$ is nef, $A=H-\varepsilon D+L-F$ is an ample $\boldsymbol{Q}$-bundle. Now, as in [F], take a

Received May 19, 1993. 
birational morphism $\pi: V^{\prime} \rightarrow V$ such that $\pi^{*} E=E_{0}+E_{1}, \pi *(\varepsilon D+G+F)=E_{0}+F_{1}$ and $E_{1} \cap F_{1}=\emptyset$. Note that $\pi_{*} E_{1} \neq 0$.

As in $[\mathrm{F}], B=F_{1}+\pi^{*} A=E_{1}+2 \pi^{*} H$ is nef as a $\boldsymbol{Q}$-bundle. Moreover, $H^{n-1} \pi^{*} E=0$ implies $H^{n-1} E_{1}=0$, so $(2 H)^{n}=(2 H)^{n-1} B$, which yields $2 H B^{n-1} \geqq B^{n}$ by the Index Theorem as in $[\mathrm{F}]$. Hence $0=B^{n-1} E_{1}=\left(\pi^{*} A\right)^{n-1} E_{1}=A^{n-1} \pi_{*} E_{1}$, contradiction.

Next we prove the following

LEMMA 2. Let $B$ be a nef and big line bundle on a normal variety $V$ with $\operatorname{dim} V=n$ and let $E=\sum \mu_{i} E_{\imath}$ be an effective divisor such that $A=B+E$ is nef. Then $h^{0}(V, A)-h^{0}(V, B) \leqq n\left(A^{n}-B^{n}\right)$.

Proof. By virtue of desingularization theory we may assume that $V$ is smooth. Let $I^{+}$be the set of $i$ 's such that $(A+B)^{n-1} E_{\imath}>0$, and set $m_{\imath}=$ $\operatorname{Max}_{a+b=n-1} A^{a} B^{b} E_{\imath}$ for each $\imath$. Since both $A$ and $B$ are nef, $m_{\imath}>0$ if and only if $i \in I^{+}$. Now we choose a sequence $S_{0}=E, S_{1}, \cdots$ of divisors in the following way: Each $S_{\jmath}$ is a $Z$-linear combination of $E_{\imath}$ 's and $S_{\jmath}=S_{\jmath-1}-E_{i(j)}$ for some $i(j)$. If $S_{\jmath-1}=\sum \nu_{i} E_{\imath}, i(j)$ is chosen from the $i$ 's where $\nu_{i} / \mu_{i}$ attains the maximum value $r_{j}$. Moreover, if $r_{j}=\nu_{i} / \mu_{i}$ for some $i \in I^{+}, i(j)$ should be chosen from such indices in $I^{+}$. Thus, finally, we have $S_{\mu}=0$ for $\mu=\Sigma \mu_{i}$. We claim $h^{0}\left(B+S_{j-1}\right)-h^{0}\left(B+S_{j}\right) \leqq n m_{i(j)}$ for any $j$.

First we consider the case $i(j) \notin I^{+}$. Then $q_{j}=\operatorname{Max}_{i \in I+\nu_{i}} / \mu_{i}<r$, by the choice of $i(j)$. We set $S_{j-1}-q_{j} E=F-G$ for some effective $\boldsymbol{Q}$-divisors $F, G$. Then $i \notin I^{+}$for any component $E_{\imath}$ of $F$, so $H_{\jmath}^{n-1} E_{\imath}=0$ for $H_{\jmath}=q_{\jmath} A+\left(1-q_{j}\right) B=$ $B+q_{j} E . \quad H_{j}$ is nef and big, and $H_{\jmath}=B+S_{\jmath_{-1}}-F+G$, hence any component of $F$ is a fixed component of $\left|B+S_{\jmath-1}\right|$ by Lemma 1 . In particular $h^{0}\left(B+S_{\jmath-1}\right)=$ $h^{0}\left(B+S_{j}\right)$, as claimed.

Next we consider the case $i(j) \in I^{+}$. Take a birational morphism $\pi: M \rightarrow E_{i(j)}$ such that $M$ is smooth and $\pi^{*}\left|\left(B+S_{\jmath-1}\right)_{E_{i(j)}}\right|=F+|H|$ with $\mathrm{Bs}|H|=\emptyset$, where $F$ is the fixed part. Let $\rho: M \rightarrow \boldsymbol{P}^{N}$ be the morphism induced by $|H|$ and let $W=\rho(M), k=\operatorname{dim} W$ and $w=\operatorname{deg} W$. Let $X$ be a general fiber of $M \rightarrow W$. Since $A+B$ is nef and big on $M$, it is so on $X$ too, hence $A^{a} B^{b} X>0$ for some $a, b$ with $a+b=\operatorname{dim} X=n-1-k$. Then $A^{a} B^{b} X \geqq 1$, so $w \leqq w A^{a} B^{b} X=A^{a} B^{b} H^{k}\{M\}$ $\leqq A^{a} B^{b} P_{j}^{k}\{M\}$ for $P_{j}=r_{j} A+\left(1-r_{j}\right) B=B+r_{j} E$, since $P_{\jmath}$ is nef and $P_{j}-H=$ $\left(r_{j} E-S_{\jmath-1}\right)+F$ is an effective $\boldsymbol{Q}$-divisor on $M$. By computation we have $\Lambda^{a} B^{b} P_{j}^{k}\{M\}=\sum_{\alpha=0}^{k}\left(\begin{array}{c}k \\ \alpha\end{array}\right) r_{j}^{\alpha}\left(1-r_{j}\right)^{k-\alpha} A^{a+\alpha} B^{b+k-\alpha}\left\{E_{i(j)}\right\} \leqq \sum_{\alpha=0}^{k}\left(\begin{array}{c}k \\ \alpha\end{array}\right) r_{j}^{\alpha}\left(1-r_{j}\right)^{k-\alpha} m_{i(j)}$ $=m_{i(j)}$, so $w \leqq m_{i(j)}$. Since $\Delta(W, \mathcal{O}(1))=k+w-N-1 \geqq 0$, we infer $h^{0}\left(E_{i(j)}, B+S_{\jmath-1}\right)$ $\leqq k+w \leqq n-1+m_{i(j)} \leqq n m_{i(j)}$. This proves the claim.

Thus the claim is established in any case. Now we infer $h^{0}(A)-h^{0}(B) \leqq$ $\sum_{\jmath}\left(h^{0}\left(B+S_{\jmath-1}\right)-h^{0}\left(B+S_{j}\right)\right) \leqq \sum_{\jmath} n m_{i(j)}=n \sum_{i} \mu_{i} m_{\imath} \leqq n \sum_{i} \mu_{i}\left(\sum_{a+b=n-1} A^{a} B^{b} E_{\imath}\right)=$ $n \sum_{a+b=n-1} A^{a} B^{b} E=n \sum_{a+b=n-1}\left(A^{a+1} B^{b}-A^{a} B^{b+1}\right)=n\left(A^{n}-B^{n}\right)$. Thus the lemma is proved. 
Proof of the Theorem. Since $\kappa(L)=n$ by assumption, there is a number $c$ such that $h^{0}(t L)>0$ for all $t \geqq c$. We will derive a contradiction assuming that $H^{n}<d-\varepsilon$ for all $\pi$ and $E$.

Replacing $\varepsilon$ if necessary, we may assume that $H_{0}^{n} \geqq d-\varepsilon-\varepsilon /(2 n)$ ! for some $H_{0}=\pi_{0}^{*} L-E_{0}, \pi_{0}: V_{0} \rightarrow V$. Let $m>0$ be an integer such that $m E_{0}$ is a $Z$-divisor and $\operatorname{Bs}\left|m H_{0}\right|=\emptyset$. Now, for any integer $s>0$, we claim $h^{0}(s m L) \leqq h^{0}\left(s m H_{0}\right)+$ $n s^{n} m^{n} \varepsilon / 2 n$ !. To see this, take a birational morphism $\pi: V^{\prime} \rightarrow V$ such that $\pi^{*}|\operatorname{sm} L|=F+|A|$ with $\mathrm{Bs}|A|=\emptyset$, where $F$ is the fixed part. We may assume that $\pi$ factors through $\pi_{0}: V_{0} \rightarrow V$ and $\left|A-\left(s m H_{0}\right)_{V^{\prime}}\right| \neq \emptyset$. Hence $h^{0}(\operatorname{smL})$ $=h^{0}(A) \leqq h^{0}\left(s m H_{0}\right)+n\left(A^{n}-s^{n} m^{n} H_{0}^{n}\right)$ by Lemma 2 . By assumption $(A / s m)^{n} \leqq$ $d-\varepsilon$, hence $A^{n}-s^{n} m^{n} H_{0}^{n} \leqq s^{n} m^{n} \varepsilon / 2 n$ !, and the claim follows.

By the hypothesis of the theorem we have a sequence $\left\{t_{j}\right\}$ such that $\lim _{\jmath \rightarrow \infty} t_{j}$ $=\infty$ and $h^{0}(V, t, L) \geqq d t_{j}^{n} / n !+\phi\left(t_{j}\right)$. For each $t_{\jmath}$, let $s$, be the least integer such that $s_{j} m \geqq t_{j}+c$. Then $h^{0}\left(t_{j} L\right) \leqq h^{0}\left(s_{j} m L\right)$, so $d t_{j}^{n} / n !+\phi\left(t_{j}\right) \leqq h^{0}\left(s_{j} m H_{0}\right)+$ $n s_{j}^{n} m^{n} \varepsilon / 2 n ! \leqq s_{j}^{n} m^{n}(d-\varepsilon) / n !+n s_{j}^{n} m^{n} \varepsilon / 2 n !+\phi\left(s_{j} m\right)$ for some polynomial $\phi$ of degree $\leqq n-1$. By the choice of $s_{j}$, we have $t_{j}>\left(s_{j}-1\right) m-c$, so $d t_{j}^{n} / n !+\phi\left(t_{j}\right)$ $=d s_{j}^{n} m^{n} / n !+\phi^{\prime}\left(s_{\jmath}\right)$ with $\lim \phi^{\prime}\left(s_{j}\right) / s_{j}^{n}=0$. Comparing the coefficients of $s_{j}^{n}$ in both sides, we get $d<d-\varepsilon+n \varepsilon / n$, a contradiction as desired.

\section{REFERENCES}

[D1] J.P. Demailly, Singular hermitian metrics on positive line bundles, Complex Algebraic Varieties; Proc. Bayreuth 1990, Lecture Notes in Math., 1507, Springer, 1992, pp. 87-104.

[D2] J.P. Demailly, A numerical criterion for very ample line bundles, J. of Diff. Geom., 37 (1993), 323-374.

[F] T. Fujita, Remarks on quasi-polarized varieties, Nagoya Math. J., 115 (1989), 105-123.

[T] H. TsuJI, Analytic Zariski decomposition I, Existence, preprint.

Department of Mathematics

Tokyo Institute of TECHNOLOGY

Oh-okayama, Meguro, Tokyo

152 JAPAN

e-mail:fujita@math.titech.ac.jp 